\title{
AEROMICOTA DE AMBIENTES INTERNOS: COMPARACION DE METODOS DE MUESTREO
}

\author{
(Aeromycota in indoor environment:sampling methods comparison)
}

\author{
Rojas, T.I.(1), Martínez, E.(2), Aira, M.J. (3)\& Almaguer, M.(1) \\ (1) Departamento de Microbiología y Virología. Facultad \\ de Biología. Universidad de La Habana (Cuba) \\ (2) Laboratorio Central de Cuarentena Vegetal \\ Centro Nacional de Sanidad Vegetal, La Habana (Cuba) \\ (3)Departamento de Botánica, Facultad de Farmacia, \\ Universidad de Santiago (España)
}

Palabras clave: Hongos, atmósfera, interiores, muestreo Key words: Fungi, atmosphere, indoor, sampling

\section{RESUMEN}

El procedimiento de muestreo utilizado para determinar el grado de contaminación fúngica en ambientes internos, difiere según los autores. Por ello, con el fin de conocer si el método utilizado por nuestro grupo de investigación, es comparable con otras metodologías, se ha realizado un estudio comparativo con varios sistemas de captación de esporas del aire, incluyendo equipos volumétricos (Aeroscope Chirana, System Air Sampler, Burkard Personal Culture) así como el método tradicional por sedimentación. Los resultados obtenidos muestran que apenas existen diferencias cuando se utilizan los sistemas volumétricos, sin embargo, el método por sedimentación ha sido menos eficaz, tanto desde el punto de vista cuantitativo como cualitativo. Mientras, el método directo por hisopado, resulta un buen complemento para determinar la fuente de contaminación. También se ha determinado la hora del día con mayor carga fúngica, que se localiza al mediodía y tras el análisis de varios medios de cultivo, se concluye que el Agar Glucosa de Sabouraud resulta adecuado para este tipo de investigaciones.

\section{INTRODUCCION}

Uno de los aspectos de interés para los investigadores que realizan estudios de aeromicología ambiental, y en concreto en el interior de edificios, es conocer si el procedimiento de trabajo se asemeja a los

Recibido el 20 Octubre 2008

Aceptado el 27 Noviembre 2008

\begin{abstract}
The sampling method used to determine the degree of fungal contamination in indoor environment differs, according to some authors. Therefore, with the purpose of knowing if the method used by our research team is comparable to other methodologies, a comparative study with various systems used to capture air spores has been carried out which include volumetric equipment (Aeroscope Chirana, System Air Sampler, Burkard Personal Culture) as well as the traditional method by sedimentation. Results have revealed that there is fairly some difference when volumetric systems are used; as to the sedimentation method, it has proved to be less effective both in the quantitative and qualitative point of view. On the other hand, direct method through sprinkling becomes a suitable tool to establish the source of contamination. Moreover it has been recorded the time of day when the greatest fungal load occurs, this being at noon so after the analysis of several culture media it has been concluded that Sabouraud Agar Glucose is suitable for this kind of research
\end{abstract}

estándares, con el fin de poder relacionar los resultados propios con los obtenidos por otros autores. En aquellos países en los que esta línea de investigación y en general, la aerobiología, está organizada en Redes de trabajo o network, son éstas las encargadas de la publicación de protocolos (Aira et al., 2005; Galán et al., 2007), lo que facilita el desarrollo de una metodología común. Sin embargo, este no siempre es el caso y los investigadores desarrollan su trabajo adaptándose a los medios disponibles, desconociendo si finalmente sus resultados 
son comparables a otros, aunque hayan sido realizados en el mismo territorio.

Los estudios sobre contaminación fúngica realizados en Cuba, en este tipo de ambientes, son cada vez más numerosos y abarcan una amplia variedad de locales (museos, oficinas, viviendas, etc.), según el objetivo particular de cada estudio (García, 1997; Fernández et al., 1998 ; Aira et al., 2002; Rojas et al., 1993, 2002; Acosta et al., 2003; Borrego et al., 2008). Por el contrario, no son muchos los trabajos que abordan el procedimiento metodológico (Rojas, 1998), por lo que en el presente estudio se incluye un análisis comparativo con diferentes métodos y equipos de muestreo, a diferentes horas del día y con varios medios de cultivo.

Para colectar las esporas del ambiente, tradicionalmente se ha usado un método gravimétrico o de sedimentación, exponiendo al aire durante un cierto tiempo una placa de Petri con medio de cultivo, pero hoy en día se prefieren los equipos de captación volumétrica que hay en el mercado, ya que a su sencillo funcionamiento, se une la ventaja de su autonomía y fácil transporte. Así mismo resulta recomendable completar este tipo de estudios con el muestreo directo (por hisopado) ya que permite identificar, con mayor precisión, la fuente de contaminación más próxima.

También la hora en que se realiza el muestreo, normalmente se acomoda a las condiciones específicas de cada estudio, sin embargo, consideramos importante determinar cuál es la más adecuada en cada caso, lo cuál estará relacionado con las condiciones climáticas (y microclimáticas) del lugar. En cuanto al medio de cultivo usado en el muestreo no hay un acuerdo unánime entre los distintos autores, por lo que se ha realizado también un estudio comparativo con los más recomendados para este tipo de investigación.

\section{MATERIALES Y METODOS}

El estudio se llevó a cabo en varios locales (museos, bibliotecas, almacenes) de la ciudad de La Habana (Cuba), sin embargo, ya que no se trata de ofrecer los resultados específicos de los mismos, sino profundizar en los aspectos metodológicos, omitiéndose su descripción por resultar irrelevante para este trabajo.

Los equipos de captación volumétrica que se han evaluado, han sido: un Aeroscope Chirana (AERO), un System Air Sampler (SAS) y un Burkard Personal Culture (BPC). Todos ellos permiten la incorporación de una placa de Petri con medio de cultivo y cada fabricante facilita instrucciones precisas, tanto en relación a su correcto manejo, como en la forma de recuento de las colonias y su transformación a unidades formadoras de colonias (ufc/ $\mathrm{m}^{3}$ ), que ha sido la expresión utilizada para cuantificar la

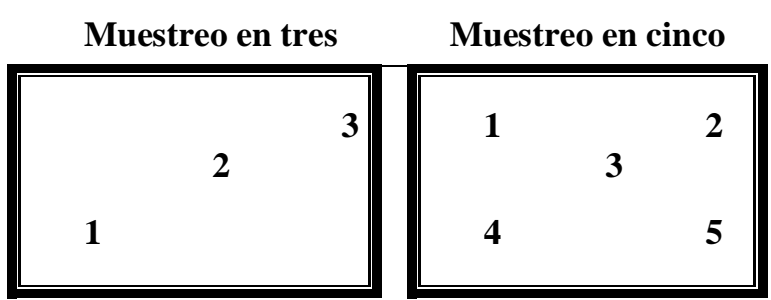

Figura 1. Esquema del diseño utilizado en los muestreos

contaminación fúngica. Por su parte, para el método gravimétrico se han seguido las recomendaciones de Omeliansky (OME) calculando el número de ufc $/ \mathrm{m}^{3}$ según el método descrito en NRP-201 (1987).

El diseño experimental para validar el método de muestreo, se enfocó para averiguar preferentemente la eficacia del equipo AERO, por ser el de uso más habitual en nuestro laboratorio. Para ello, se realizaron dos muestreos en dos locales diferentes, combinando el uso de dicho equipo con los demás. En todos los muestreos se siguió un diseño diagonal tomando 3 ó 5 puntos de acuerdo a la superficie de los mismos, según Norma FEDECAI-01 (2007), tal como se señala en la Figura 1, y realizando tres réplicas en cada punto.

Además del muestreo ambiental realizado por captación de esporas del ambiente, se han tomado muestras por el método de hisopado (NRP-201, 1987) a los sustratos y/o objetos presentes en el interior de los locales. En ambos casos, y con el fin de valorar las posibles variaciones entre ambos métodos, se procedió al estudio de las colonias, identificando a nivel de género de acuerdo a Barnett \& Hunter (1998). Para los géneros más abundantes se siguieron los criterios de identificación de Ellis (1971, 1976), Klich \& Pitt (1994), Ho et al. (1999) y Pitt (2000).

En cuanto a los medios de cultivo, se han valorado los cuatro siguientes, M1 (Agar Extracto de Malta suplementado con peptona y glucosa (ACGIH, 1989); M2 (Agar Extracto de Malta+7,5\% $\mathrm{NaCl}$ ); M3 (Agar Papa Dextrosa +7,5\% NaCl) y M4 (Agar Glucosa de Sabouraud) y el estudio en el tiempo se realizó muestreando cada hora entre las 7 a.m. y las 4 p.m.

Los resultados obtenidos se han valorado estadísticamente, aplicando el test de Students, la Prueba de Kruskall-Wallis y/o la prueba de Scheffé del paquete informático StatSoft, Inc. (2001).

\section{RESULTADOS Y DISCUSION}

El sistema AERO se probó frente a los dos sistemas volumétricos (SAS y BPC) y el método gravimétrico (OME) en dos locales diferentes (L1 y L2). Los resultados 
muestran que no existen diferencias significativas en cuanto a los niveles de contaminación fúngica, en el primer caso, mientras que al comparar AERO con OME, el sistema volumétrico se muestra mucho más eficaz, ya que recoge prácticamente el doble de unidades formadoras de colonias (Figura 2).

Estos resultados coinciden con otros autores que señalan que el método por sedimentación en placas no permite comparar de forma favorable los resultados obtenidos por otros métodos de muestreo (Buttner \& Stetzenbach, 1993; Caneva et al., 2004).

Los equipos volumétricos SAS y BPC, son utilizados con frecuencia en estudios aerobiológicos (Bellin \& Schillinger, 2001; Aira et al., 2007) debido, entre otras ventajas, a su poco peso en comparación con el equipo AERO. Pese a ello, dicho equipo es utilizado de manera frecuente, tanto en ambientes de interior (Blomquist et al., 1984; Klanova, 2000) como de exterior (Medrela- Kuder,

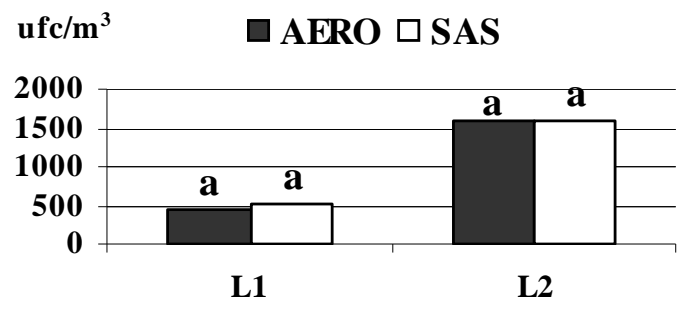

\section{$\mathrm{uf} / \mathrm{cm}^{3} \quad \square$ AERO $\square$ OME}
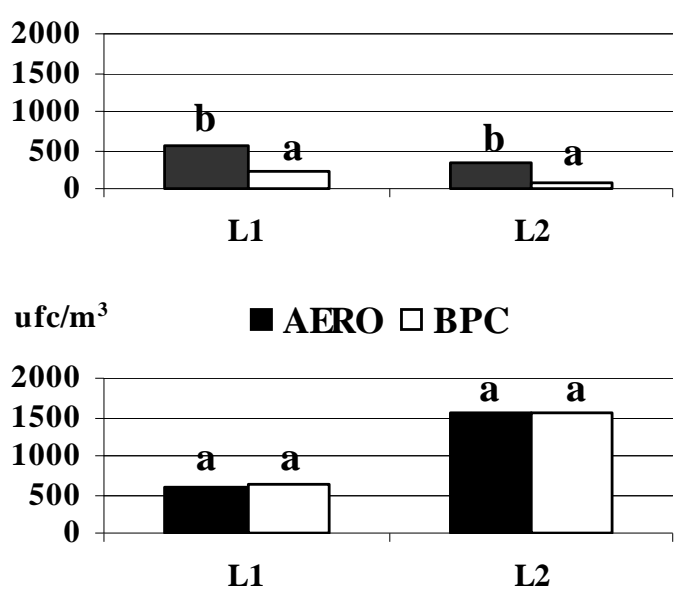

Leyenda.-AERO (Aeroscopio Chirana),

SAS (Sistem Air Sampler),

BPC (Burkard Personal Culture),

OME (Omeliansky),

L1 y L2 (locales de muestreo)

Figura 2. Valores promedios de ufc/ $/ \mathrm{m}^{3}$ obtenidos en la comparación de diferentes métodos de muestreo (letras no comunes indican diferencias significativas de $p<0,05$, según test de Students)
2003) y algunos autores le reconocen ciertas ventajas con respecto a otros modelos; a señalar la homogénea distribución de las esporas en la placa (debido a la rotación de ésta bajo la hendidura) y la posibilidad de poder usar diferente volumen de aire dependiendo de la anchura de la rejilla utilizada, lo cual facilita seleccionar la misma de acuerdo a los niveles esperados (Portnoy et al., 2004).

Mas recientemente se han desarrollado nuevos modelos, basados en el mismo principio que el equipo AERO (STA-100, 203 y 204, entre otros), los cuales son utilizados con frecuencia en estudios ambientales de edificios, industria farmacéutica, hospitales, laboratorios de cosméticos y de alimentos (Horn, 2005).

Para evaluar la eficacia del equipo AERO, esta vez desde el punto de vista cualitativo, se realizó un nuevo muestreo para compararlo con SAS y OME, identificando los aislamientos a nivel de género. Los resultados obtenidos (Figura 3) muestran que los géneros Aspergillus, Cladosporium y Penicillium fueron los más abundantes en cuanto a unidades formadoras de colonias, no presentándose tampoco diferencias significativas entre ambos sistemas volumétricos, pero sí entre el equipo AERO y el método OME, tanto para Aspergillus como para Penicillium.

El hecho de que dichas diferencias no afecten al género Cladosporium, lo atribuimos a la dimensión de los conidios que son más grandes que los de Aspergillus y Penicillium y por tanto, tienen un mayor tamaño aerodinámico, ya que de acuerdo con Caneva et al. (2004), la micobiota del aire colectada dependerá, entre otros factores, del tamaño y forma de las partículas, del movimiento del aire circundante, mientras la sedimentación no favorece la representación de hongos con esporas pequeñas.

En el mismo sentido, Pasanen et al. (1991), señalan que en la depositación de las esporas hay que tener en cuenta el tamaño aerodinámico, que aumentará con el incremento de la humedad relativa del aire, el que favorecerá la rápida caída de las partículas de mayor tamaño. Por lo tanto, teniendo en cuenta los resultados obtenidos con el Aeroscope Chirana, coincidimos con lo señalado por Burge \& Solomon (1987) en que este método es eficiente en la colecta de microorganismos ambientales.

$\mathrm{Al}$ representar las especies más abundantes en los locales muestreados, a partir de las esporas del ambiente (con el equipo AERO) o de las recogidas por hisopado (Tabla 1), se observan diferencias considerables, tanto en el número de especies identificadas (28 ambiente vs. 22 por hisopado) como en su frecuencia relativa. Así especies como Aspergillus flavus, A.niger, Cladosporium cladosporioides, C. sphaerospermum y Penicillium citrinum, son ubicuas en todos los locales muestreados mientras en las muestras de hisopado su frecuencia relativa oscila 


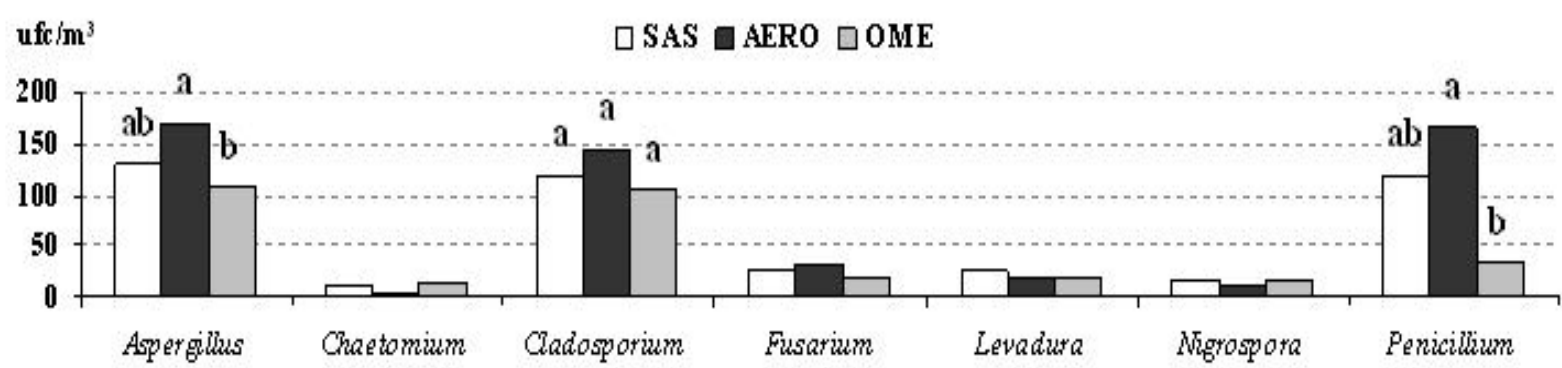

Figura 3. Valores promedios de ufc/ $\mathbf{m}^{3}$ de los géneros identificados con diferentes métodos de muestreo (letras no comunes indican diferencias significativas de p<0,05, según Prueba de Kruskall-Wallis para Cladosporium y prueba de Scheffé para Aspergillus y Penicillium

entre un $17-50 \%$. Por su parte, Aspergillus tamarii y Cladosporium herbarum tienen una mayor presencia en los sustratos muestreados que en el aire. Estos resultados pueden ser útiles para localizar la fuente de contaminación del local y valorar la influencia del aporte del aire exterior en aquellos que tengan comunicación con el exterior.

La evaluación del medio de cultivo también se realizó desde un doble punto de vista, cuantitativo y cualitativo. En el primer aspecto, se observa que el medio M2 logró la mayor eficiencia aunque no muestra diferencia significativa desde el punto de vista estadístico con M3, pero sí con M1 y M4. El medio M3 no difiere con ninguno de los medios evaluados (Figura 4).

Se debe señalar que $\mathrm{M} 1$ es el medio recomendado para este tipo de estudios según la ACGIH (1989), el cual presentó los valores más bajos de recuperación y ninguna diferencia significativa desde el punto de vista estadístico con M4.

Para evaluar los resultados desde el punto de vista cualitativo, se identificaron los aislamientos realizados a nivel de género. En la Figura 5 se observa que

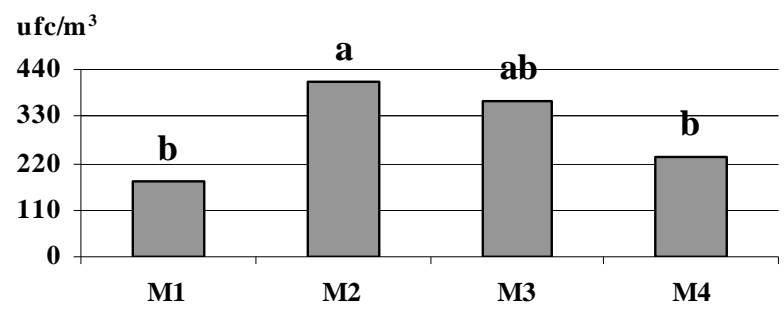

M1 (agar extracto de malta suplementado con peptona y glucosa), M2 (agar extracto de malta+7.5\% NaCl), M3 (agar papa dextrosa $+7.5 \% \mathrm{NaCl}$ ), $\mathbf{M} 4$ (agar glucosa de Sabouraud)

Figura 4. Valores promedios de ufc/ $\mathbf{m}^{3}$ obtenidas con Aeroscope Chirana en diferentes medios de cultivos (letras no comunes indican diferencias significativas, según Prueba de Scheffé para p<0,05) los géneros Aspergillus, Penicillium y Cladosporium fueron los más abundantes, independientemente del medio

Tabla 1. Comparación de la frecuencia relativa (F.R.\%) de las especies identificadas en el ambiente y en los

\begin{tabular}{|l|c|c|}
\hline & $\begin{array}{c}\text { AERO } \\
\text { (F.R. \%) }\end{array}$ & $\begin{array}{c}\text { Hisopado } \\
\text { (F.R\%) }\end{array}$ \\
\hline Aspergillus caespitosus & 11 & 8 \\
\hline Aspergillus candidus & 33 & 16 \\
\hline Aspergillus clavatus & 33 & 16 \\
\hline Aspergillus flavus & 100 & 50 \\
\hline Aspergillus fumigatus & 55 & 25 \\
\hline Aspergillus niger & 100 & 42 \\
\hline Aspergillus ochraceus & 11 & -- \\
\hline Aspergillus oryzae & 44 & 25 \\
\hline Aspergillus parasiticus & 11 & -- \\
\hline Aspergillus puniceus & 11 & -- \\
\hline Aspergillus scleriotorum & 22 & -- \\
\hline Aspergillus sydowii & 33 & 25 \\
\hline Aspergillus tamarii & 22 & 42 \\
\hline Aspergillus terreus & 67 & 33 \\
\hline Aspergillus ustus & 22 & -- \\
\hline Aspergillus wentii & 22 & -- \\
\hline Aspergillus versicolor & 55 & 25 \\
\hline Cladosporium cladosporioides & 100 & 42 \\
\hline Cladosporium herbarum & 14 & 17 \\
\hline Cladosporium oxysporum & 71 & 33 \\
\hline Cladosporium sphaerospermum & 100 & 17 \\
\hline Penicillium chrysogenum & 86 & 50 \\
\hline Penicillium citrinum & 100 & 17 \\
\hline Penicillium corylophylum & 29 & 17 \\
\hline Penicillium glabrum & 71 & 33 \\
\hline Penicillium janthinellum & 14 & 8 \\
\hline Penicillium purpurogenum & 71 & 8 \\
\hline Penicillium simplicissimun & 71 & 8 \\
\hline & & \\
\hline
\end{tabular}




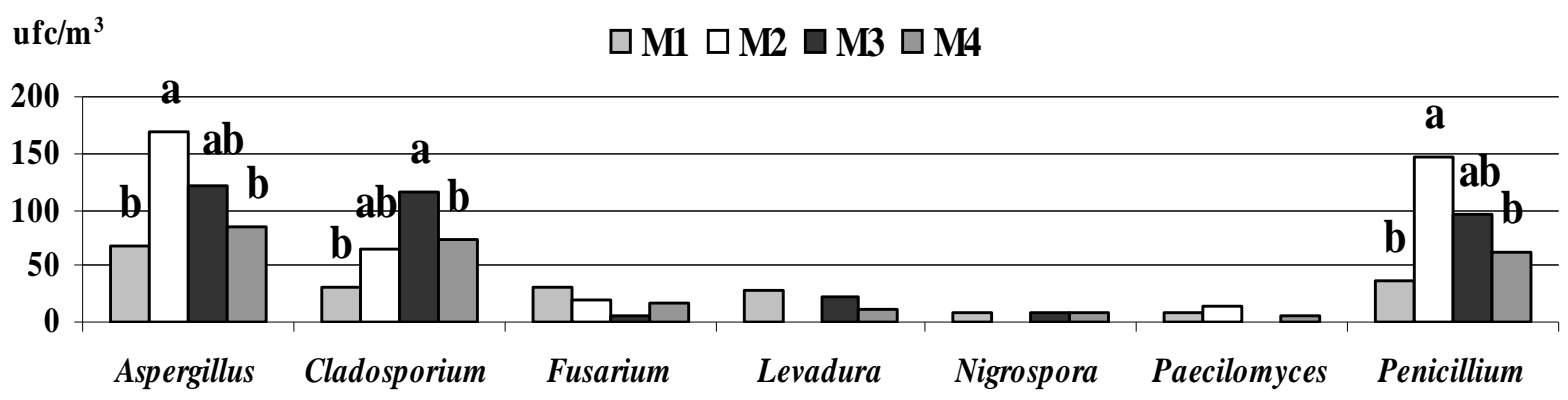

Figura 5. Niveles promedios de $u f c / \mathbf{m}^{3}$ de hongos y levaduras en diferentes medios de cultivo (letras no comunes indican diferencias significativas, según Prueba de Scheffe para p<0,05)

de cultivo, resultando más eficaz M2 para Aspergillus y Penicillium y M3 para Cladosporium.

Ambos medios no presentaron diferencias en cuanto a la significación estadística para la colecta de los tres géneros evaluados. El resto de los medios (M1 y M4) no presentan diferencias entre sí y difieren de M2. Sin embargo, atendiendo a la diversidad que es capaz de recuperar cada uno de los medios, M1 y M4 son los más adecuados ya que en M2 no se representaron las levaduras ni los géneros Paecilomyces ni Nigrospora, y en M3 no se obtuvo Paecilomyces y se recuperan bajos niveles de Fusarium.

Teniendo en cuenta los aspectos anteriores, podría recomendarse el uso de M4 (agar glucosa de Sabouraud), ya que aunque no es el medio donde se obtiene los mayores niveles, los valores obtenidos están en el mismo orden $\left(10^{2}\right)$ que los de M2 y M3 y es el medio que conjuntamente con M1 detecta la mayor variabilidad fúngica del ambiente. Además su eficiencia en la recuperación y variabilidad obtenida no difieren de las de M1 (medio recomendado por la ACGIH, 1989).

Al revisar la bibliografía relativa a este tema, se concluye que no hay un acuerdo, ya que para algunos autores el Agar Extracto de Malta +7,5\% NaCl (M2), no es adecuado para promover la esporulación (Morring et al., 1983; Verhoeff et al., 1990), recomendándose suplementarlo con peptona y glucosa (Hunter et al., 1988; ACGIH, 1989) o usar Agar glucosa de Sabouraud (Medina et al., 1999; Pepeljnjak \& Segvic, 2003).

Finalmente para averiguar si pueden ocurrir variaciones significativas según el momento en que se recogen las muestras, se realizó un muestreo cada hora entre las 7a.m. y las 4 p.m. en los dos locales (L1 y L2). Los resultados obtenidos (Figura 6) señalan que las horas en las que se alcanza un mayor nivel de contaminación son las 7, las 12 y las $11 \mathrm{~h}$. (L1) y las 11, 12 y $14 \mathrm{~h}$ (L2), aunque no se encontraron diferencias significativas entre ellas (Tabla 2).

Muchos hongos liberan sus esporas durante la noche y primeras horas de la mañana favoreciendo a este proceso la elevada humedad un aspecto que justifica que encontremos picos de máxima concentración a las 7 de la mañana ya que las ventanas del Local (L1) permanecen abiertas durante la noche.

De todas formas, al calcular los valores promedio de contaminación en ambos muestreos, la hora de mayor concentración fúngica resultó las $12 \mathrm{~h}$, con $3327 \mathrm{ufc} / \mathrm{m}^{3}$, con valores muy próximos a los obtenidos a las 11h (3086 $\mathrm{ufc} / \mathrm{m}^{3}$ ), por lo que esta franja horaria podría ser la más conveniente. Este comportamiento coincide con los resultados obtenidos por Shadzi et al. (1993), al estudiar zonas urbanas de Irán durante un año, en las cuales encontraron que la mayor concentración de hongos se presentó en el mediodía y al final de la mañana y con el patrón de periodicidad diurna descrito para Cladosporium, por Shenoi \& Ramalingam (1975) quienes señalan que durante las horas del mediodía sus niveles son mayores.

$\mathbf{u f c} / \mathbf{m}^{3}$

L1

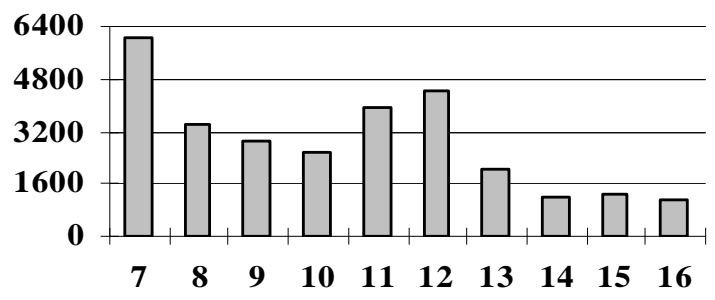

$\mathbf{u f c} / \mathbf{m}^{3}$

L2

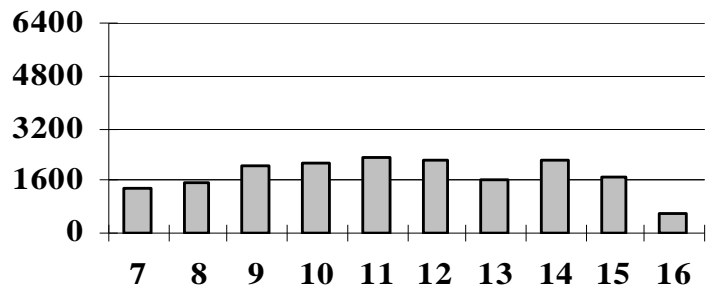

Figura 6.- Niveles promedios de ufc/m $\mathrm{m}^{3}(\mathrm{~L} 1$ y L2, locales de muestreo) 
Tabla 2. Comparación de ufc. $\mathrm{m}^{-3}$ en ambos locales (L1 y L2) a diferentes horas (letras no comunes indican diferencias significativas, según Prueba de Scheffé para $\mathrm{p}<0,05$ )

\begin{tabular}{|c|c|c|}
\hline \multicolumn{3}{|c|}{ L 1} \\
\hline Hora & ufc/m $/ \mathbf{m}^{3}$ & Significación \\
\hline 7 & 6053 & a \\
\hline 12 & 4428 & $\mathrm{ab}$ \\
\hline 11 & 3904 & abc \\
\hline 8 & 3445 & abcd \\
\hline 9 & 2882 & bcd \\
\hline 10 & 2538 & bcd \\
\hline 13 & 2027 & bcd \\
\hline 15 & 1314 & $\mathrm{~cd}$ \\
\hline 14 & 1213 & $\mathrm{~cd}$ \\
\hline 16 & 1102 & $\mathrm{~d}$ \\
\hline \multicolumn{3}{|c|}{ L 2} \\
\hline Hora & ufc $/ \mathrm{m}^{3}$ & \begin{tabular}{|l|} 
Significación \\
\end{tabular} \\
\hline 11 & 2268 & $\mathrm{a}$ \\
\hline 14 & 2250 & $\mathrm{a}$ \\
\hline 12 & 2226 & $\mathrm{a}$ \\
\hline 10 & 2115 & $\mathrm{a}$ \\
\hline 9 & 2065 & $\mathrm{a}$ \\
\hline 15 & 1679 & $\mathrm{ab}$ \\
\hline 13 & 1646 & $\mathrm{ab}$ \\
\hline 8 & 1531 & $a b$ \\
\hline 7 & 1357 & $a b$ \\
\hline 16 & 607 & b \\
\hline
\end{tabular}

Ricci et al. (1996), señalaron que las concentraciones de esporas fúngicas en ambientes de interior son influenciadas por parámetros ambientales (temperatura, humedad, corrientes de aire, etc), características del local (sustratos, ventilación, limpieza, etc.) siendo también una variable importante la micobiota predominante en el aire exterior (Shelton et al., 2002).

\section{REFERENCIAS}

ACGIH (1989). Guidelines for the assessment of bioaerosols in the indoor environment. Cincinnati, American Conference of Governmental Industrial Hygienists, 110p.

Acosta, S.; Rojas, T.I.; González, F.; Galán, I.; Benítez, M.; Llanes, N.; Sánchez, V.; Hernández, D. \& Casadesús, L. (2003). Caracterización microbiológica del ambiente y de los materiales de un almacén soterrado. $2^{\text {da }}$ Jornada científica y de calidad. CEINPET

Aira, M.J.; Rojas, T.I. \& Jato, V. (2002). Fungi associated with three houses in Havana (Cuba). Grana 41:114-118

Aira, M.J.; Jato, V.; Iglesias; Rodríguez-Rajo, F.J.; Méndez, J.; Albelda, Y.; Hervés, M.; Piontelli, E. \& Stchigel, A.M. (2005). Calidad del aire. Polen y esporas en la comunidad gallega. Xunta de Galicia.

Aira, M.J.; Jato, V.; Stchigel, A.M.; Rodríguez-Rajo, F.J. \& Piontelli, E. (2007). Aeromicological study in the Cathedral of Santiago de Compostela (Spain). International Biodeterioration \& Biodegradation 60:231-237

Barnett, H. L. \& Hunter, B. B. (1998). Illustrated Genera of Imperfect Fungi, Fourth Edition APS Press. The American Phytopathological Society, USA.

Bellin, P. \& Schillinger, J. (2001). Comparison of field performance of the Andersen N6 single stage and the SAS sampler for airborne fungal propagules. Indoor Air 11:65-68

Blomquist, G.; Ström, G.; Stromquist, L.H. (1984). Sampling of high concentrations of airborne fungi. Scand. J. Work Environ. Health. 10:109-113

Borrego, S.; Pons, V.; Perdomo, I. (2008). Estudio de la contaminación microbiana del aire en dos depósitos del Archivo Nacional de la República de Cuba. Revista CNIC Ciencias Biológicas

Burge, H. A. \& Solomon, W.R. (1987). Sampling and analysis of biological aerosols. Atmos. Environ. 21:451-456

Buttner, P.M. \& Stetzenbach, L.D. (1993). Monitoring airborne fungal spores in an experimental indoor environment to evaluate sampling methods and the effects of human activity on air sampling. Appl. Environ. Microbiol. 59:219-226

Caneva, G.; Nugari, M.P.; Romolaccio, M.; Zuccarello, V. (2004). Aerobiology in museums comparison between sampling methods. Proceeding the XI International Palynological Congress, pp.87-88.

Ellis, M.B. (1971). Dematiaceous hyphomycetes. Commonwealth Mycological Institute, Kew, Sourvey, England, CMI.

Ellis, M.B. (1976). More Dematiaceous Hyphomycetes. Comm. Mycol. Inst. Kew. England.

FEDECAI-01 (2007). Programa de certificación de calidad ambiental en interiores. Calidad ambiental en interiores: Criterios de muestreo. pp 4-6. Federación Española de Empresas de Calidad Ambiental Interior (FEDECAI).

Fernández, M.; Cabrera, E.; Rojas, T.I \& Fernández, F. (1998). Estudio de la microflora existente en almacenes textiles, bajo condiciones de clima tropical. Boletín INTEXTER 114:49-54

Galán, C.; Cariñanos, P.; Alcazar, P.; Domínguez, E.; Aira, M.J. et al. (2007). Manual de Calidad y Gestión de la Red Española de Aerobiología. Servicio de Publicaciones. Universidad de Córdoba.

García, T. (1997). Consideraciones sobre la micobiota de la mapoteca del Archivo Nacional. Trabajo de Diploma. Facultad de Biología. Universidad de La Habana.

Ho, M.H.M.; Castañeda R.F.; Dugan F.M. \& Jong S.C. (1999). Cladosporium and Cladophialophora in culture: Descriptions and an expanded key. Mycotaxon LXXII:115-157 
Horn, J. (2005). Air samplers for environmental monitoring regarding ISO 14698. Newsletter No. 65

Hunter, C.A.; Grant, C.; Flannigan, B.; Bravery, A.F. (1988), Mould in buildings: the air spore of domestic dwellings. J. Int. Biodet. 24:81-101

Klanova, K. (2000). The concentrations of mixed populations of fungi in indoor air: rooms with and without mould problems and rooms with and without health complaints. Cent. Eur. J. Public. Health. 8:59-61

Klich, M.A. \& Pitt, J.I. (1994). A Laboratory guide to common Aspergillus species and their teleomorphs. Comm. Sc. and Industrial R. Org.

Medina, L.; Tuozzo, A.; Herrera, J.; Perozo, Y.; González, L. (1999). Estudio de hongos en bibliotecas de la Universidad de Carabobo-Valencia (Venezuela), Revista Universidad 3:5-20

Medrela-Kuder, E. (2003). Seasonal variations in the occurrence of culturable airborne fungi in outdoor and indoor air in Craców. International Biodeterioration \& Biodegradation 52:203-205

Morring, K.L.; Sorenson, W.G. \& Attfield, M.D. (1983) Sampling for airborne fungi: A statistical comparison of media. Am. Ind. Hyg. Assoc. J., 44:662-664

NRP-201 (1987). Análisis Ambiental. Método de Omeliansky. Análisis higiénico sanitario y ambiental. Métodos de ensayos microbiológicos. Norma Ramal de la Pesca. Ministerio de la Industria Pesquera.

Pasanen, A.L.; Pasanen, P.; Jantunen, M.J. \& Kalliokoski, P. (1991). Significance of air humidity and air velocity for fungal spore release into the air. Atmospheric Enviroment 25:459-462

Pepeljnjak, S. \& Segvic, M. (2003). Occurence of fungi in air and on plants in vegetation of different climatic regions in Croatia. Aerobiologia, 19:11-19

Pitt, J.I. (2000). A laboratory guide to common Penicillium species. Food Science Australia. Third Edition.
Portnoy, J.M.; Barnes, C.S. \& Kennedy, K (2004). Sampling for indoor fungi. Current reviews of allergy and clinical immunology. Journal Allergy Clin Immunol 113:189-198

Ricci, S; Bruni, M; Meriggi, A \& Corsico, R. (1996). Aerobiological monitoring for fungal spore in rehabilitation hospital in northem Italy. Aerobiología 12:233-237

Rojas, T. I. (1998). Micobiota contaminante en ambientes interiores. Tesis de Maestría. Facultad de Biología, Universidad de la Habana.

Rojas, T.I.; Cruz, I. \& Alvarado, Y. (1993). Hongos filamentosos como contaminantes ambientales en locales de almacenamiento. IV Simposio de Botánica. I Simposio Latinoamericano de Micología. Palacio de las Convenciones. La Habana, 22-26 de junio

Rojas T.I.; Martínez, E.; Gómez, Y. \& Alvarado, Y. (2002). Airborne spore of Aspergillus in cultural institutions at Havana University. Grana 41:190-193

Shadzi, S.; Zahraee, M.H. \& Chadeganipour, M. (1993). Incidence of airborne fungi in Isfahan, Iran. Mycoses 36:69-73

Shelton, B.G.; Kirland, K.H.; Flanders, W.D. \& Morris, G.K. (2002). Profiles of airborne fungi in buildings and outdoor environments in the United States. Applied and Environment Microbiology 68:1743-1753

Shenoi, M. M. \& Ramalingam, A. (1975). Circadian periodicities of some spore components of air of Mysore Arogya. J. Health Sci. $1: 154-156$

StatSoft, Inc. (2001): Statistica (data analysis software system), version 6 . www.statsoft.com.

Verhoeff A.P.; van Wijnen, J.I.; Boleij, J.S.M. \& Brunekreef, B. (1990). Enumeration and identification of airborne viable mould propagules in houses. Allergy 45:275-84 